\title{
Revue Française de Civilisation Britannique
}

French Journal of British Studies

$\mathrm{XII-2}$ | 2003

La situation et les politiques de l'emploi en France et en Grande-Bretagne, 1990-2000

\section{Les politiques de retour à l'emploi en Grande- Bretagne et en France : éléments pour une comparaison}

Welfare to Work Policies in Britain and France: Some Comparative Elements

Jean-Paul Révauger

\section{OpenEdition}

Journals

Édition électronique

URL : http://journals.openedition.org/rfcb/3152

DOI : $10.4000 /$ rfcb.3152

ISSN : 2429-4373

Éditeur

CRECIB - Centre de recherche et d'études en civilisation britannique

Édition imprimée

Date de publication : 1 avril 2003

ISBN : 2-911580-16-8

ISSN : 0248-9015

Référence électronique

Jean-Paul Révauger, « Les politiques de retour à l'emploi en Grande-Bretagne et en France : éléments pour une comparaison », Revue Française de Civilisation Britannique [En ligne], XII-2 I 2003, mis en ligne le 01 avril 2003, consulté le 31 mai 2019. URL : http://journals.openedition.org/rfcb/3152 ; DOI :

$10.4000 /$ rfcb.3152

Ce document a été généré automatiquement le 31 mai 2019.

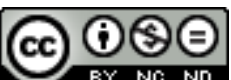

Revue française de civilisation britannique est mis à disposition selon les termes de la licence Creative Commons Attribution - Pas d'Utilisation Commerciale - Pas de Modification 4.0 International. 


\title{
Les politiques de retour à l'emploi en Grande-Bretagne et en France : éléments pour une comparaison
}

\author{
Welfare to Work Policies in Britain and France: Some Comparative Elements
}

Jean-Paul Révauger

1 Une comparaison entre les politiques de l'emploi en Grande-Bretagne et en France fait apparaître d'une part des différences historiques sensibles et d'autre part des convergences réelles dans les politiques publiques, et même les concepts utilisés pour penser la question.

2 Historiquement, tant les conditions objectives que le rythme de la politique économique ont été dissemblables. Il n'est pas indifférent à notre propos que la Grande-Bretagne ait été un pays totalement dominé par l'industrie au $\mathrm{XX}^{\mathrm{e}}$ siècle, alors que l'agriculture, puis les services occupaient une place relativement plus grande en France. Le choc de la révolution technologique et le passage à une économie post-industrielle, dans les années 1980, ne pouvaient être, mécaniquement, que beaucoup plus brutaux en Grande-Bretagne qu'en France, indépendamment des politiques publiques suivies.

3 Celles-ci ont également connu un rythme différent. La pensée économique des gouvernements français et les discours dominants sont restés influencés par le keynésianisme jusqu'en 1982. Le "plan Barre» adopté sous la présidence de Valéry Giscard d'Estaing, comme la politique de relance par la consommation et la revalorisation des retraites suivie sous celle de François Mitterrand jusqu'au tournant de la rigueur, pris officiellement en 1983, mais sensible dès 1982, puisaient à la même source. En GrandeBretagne, en revanche, Edward Heath semble bien avoir été le dernier premier ministre keynésien. Ce sont les travaillistes qui abandonnent officiellement le keynésianisme, à partir de 1975, non sans laisser dans l'histoire quelques fortes maximes, telles que «the party is over ». ${ }^{1}$ Il va de soi que l'abandon du keynésianisme, pour lequel le plein emploi était la priorité, n’a pas été sans conséquences sur le niveau de chômage et sur les politiques d'emploi. ${ }^{2}$. 


\section{Un décalage temporel dans les politiques de l'emploi}

Seconde différence dans l'histoire des politiques publiques : les politiques d'emploi à proprement parler connaissent dans les deux pays une rupture, mais celle-ci est décalée d'à peu près onze ans. Elle intervient en 1979 en Grande-Bretagne, date à laquelle Margaret Thatcher accède au pouvoir. Pendant les premières années de la décennie Thatcher, le chômage sera présenté de façon très surprenante pour les observateurs extérieurs. Le chômage est alors perçu comme un phénomène naturel, dont la cause principale est la cherté du travail. Le syndicalisme est mis à l'index, car il est accusé d'avoir contribué à augmenter le coût du travail, et donc, le chômage. Un économiste thatchérien en vue, Patrick Minford, n'hésitera pas à venir expliquer aux universitaires français que si le chômage, à court terme, permet de réduire le pouvoir syndical, il aura un effet bénéfique à long terme sur l'économie. La crise était donc grosse d'un monde nouveau, et les destructions portaient en elles-mêmes le germe des créations de demain, en termes schumpéteriens. Aucun soutien ne sera apporté aux "lame ducks ", ces secteurs économiques en difficulté, parfois maintenus à bout de bras par les travaillistes dans la Grande-Bretagne des années 1970. La réduction du taux d'inflation, et la marche en avant de la nouvelle économie ne devaient pas être entravées par des politiques coûteuses et contre-productives. Les conditions d'attribution des allocations-chômage furent donc sévèrement durcies, et leur montant réduit tout au long de la décennie, le chômeur devant démontrer qu'il était « disponible pour le travail ». ${ }^{3}$ Les chiffres du chômage (3 millions en 1982), bien qu'impressionnants, masquent en réalité l'étendue des changements dans le marché du travail. Le nombre de postes de travail détruits dans le domaine industriel et créés dans celui des services est bien plus considérable encore. Le chômage avait donc une fonction, celle d'aiguillonner les Britanniques vers les emplois disponibles, vers la nouvelle économie. Le discours sur la fraude, sur la stigmatisation des chômeurs, est bien sûr emprunté à l'histoire de la politique sociale victorienne et profondément ancré dans une culture modelée par l'éthique protestante du travail. Mais il n'est pas irrationnel ou purement affectif, contrairement à ce que suggérait Denis Healey, qui traitait les Thatchériens de «sado-monétaristes». Toutes proportions gardées, on peut tout à fait comparer la stigmatisation du chômage dans les années 1980 à celle des pauvres dans les années 1830. Dans les deux cas, il s'agissait de faire entrer le pays dans un nouveau mode de production, industriel au XIX ${ }^{e}$ siècle, post-industriel à la fin du XXe . Les changements intervenus sous John Major sont modestes, par comparaison avec ceux du début des années 1980. Le retour des travaillistes aux affaires en 1997 se fait dans un contexte différent, c'est-à-dire après que la transition économique et technologique eut été effectuée. La comparaison avec la France devient alors d'autant plus pertinente que celle-ci se retrouve, à la même époque, dans une situation beaucoup plus proche de celle de sa voisine, après avoir emprunté des chemins différents du point de vue des politiques d'emploi.

5 En effet, en France, ces politiques d'emploi ne connaissent de rupture qu'en 1990. Avant cette date, la montée du chômage de masse est perçue comme une évolution dramatique appelant avant tout un traitement social, plutôt qu'un traitement économique. Le fait que le passage du fordisme au post-fordisme se fasse dans ce pays sous des gouvernements à direction socialiste n'est pas indifférent. Globalement, et malgré quelques débats, l'ensemble de la gauche française, y compris le parti communiste, accepta après 1983 
l'idée que l'État ne jouissait pas d'une marge de manœuvre très grande en matière de politique économique, et que ce que l'on appellera plus tard la mondialisation interdisait une politique de relance dans un seul pays. Le mythe mobilisateur du "droit au travail ", idée force de la révolution de 1848 miraculeusement rendue possible par la grâce conjointe de John Maynard Keynes et de Henry Ford, céda donc peu à peu la place à l'accompagnement social des restructurations, non sans laisser beaucoup d'amertume ${ }^{4}$. Les autorités ciblèrent leur politique en direction de trois publics, les chômeurs âgés, les jeunes, et les chômeurs de longue durée. La solution trouvée au problème des travailleurs âgés ayant perdu leur emploi ou sur le point de le perdre est sans équivalent à l'extérieur du pays. Il s'agit bien sûr des préretraites, dont le poids économique et l'impact furent considérables, y compris sur le plan idéologique et politique. Dès 1983, les préretraités représentaient $60 \%$ des bénéficiaires des politiques d'emploi. ${ }^{5}$ En 1984, il fut décidé de ne plus faire supporter le coût des préretraites à la seule Unedic. Celui-ci fut dès lors pris en charge à $70 \%$ par l'État, sauf dans le cas des chômeurs de plus de 55 ans que l'on se contentait de dispenser de rechercher un emploi, et qui étaient donc définitivement au chômage en attendant la retraite. On passa donc d'un financement par l'assurance à un financement par l'assistance, qui sera peu à peu appelée "solidarité », terme moins stigmatisant. On assiste donc, dans les deux pays, à un basculement du financement du chômage. En revanche, la Grande-Bretagne n'a pas l'équivalent des préretraites françaises. Le taux d'activité des hommes de 55 à 64 ans passa en France de 69,9\% en 1979 à $53,6 \%$ en 1983 , puis $45,8 \%$ en 1990 et $41,5 \%$ en 1995 . La Grande-Bretagne connut une évolution beaucoup moins spectaculaire, avec $71,5 \%$ en $1983,68,1 \%$ en 1990 et $62,5 \%$ en $1995 .{ }^{6}$

6 Le coût budgétaire était colossal, mais ce système présentait l'avantage pour l'État d'étaler les coûts des restructurations sur au moins cinq ans, parfois beaucoup plus dans les cas extrêmes comme la sidérurgie, où des préretraites furent accordées à 52 ans. Le manque à gagner pour les régimes de retraites était également très important. Les avantages étaient aussi évidents pour les bénéficiaires, qui jouissaient ainsi de revenus stables sans être astreints à travailler, et pour les employeurs qui se séparaient d'une main-d'œuvre onéreuse, du fait de son ancienneté, et réputée plus difficile à former aux nouvelles technologies et aux nouvelles formes du travail. La problématique, très présente aux États-Unis, de la stigmatisation des personnes âgées, connue sous le terme d' ' ageism» a eu un impact faible en France. ${ }^{7}$ Les préretraites étaient en phase avec une vieille culture syndicale, pour laquelle "il vaut mieux payer des retraités que des chômeurs ", et ne manquèrent pas d'influencer le débat sur la durée du travail. Le partage du travail et la valorisation de la libération du temps sont un des termes de la complexe équation des 35 heures, qui rassemble toutes les ambiguïtés françaises par rapport au travail.

7 La situation des chômeurs de longue durée déboucha entre autres choses sur la création du RMI en 1988, c'est-à-dire, une fois encore, sur une prise en charge du chômage par la "solidarité " et non plus par l' "assurance ». La majorité des RMIstes, dont le nombre s'est élevé jusqu'à un million, se révélèrent être des chômeurs en fin de droits. ${ }^{8}$ Un débat intense se déroula à l'Assemblée nationale, reflétant celui qui oppose partisans d'un revenu de citoyenneté sans contrepartie et partisans d'un système inspiré par le workfare américain. Cela déboucha sur un compromis, la notion d'insertion, qui accéda par la suite au rang de concept structurant de la politique sociale française. ${ }^{9}$ Ce compromis fait partager en théorie le fardeau à la collectivité, censée offrir de réelles occasions 
d'insertion aux allocataires, et à ces derniers, censés fournir les efforts nécessaires pour retrouver un emploi ou améliorer leur situation de quelque autre manière. En réalité, il faut attendre l'an 2000 pour que la réalité de l'insertion et de la formation des chômeurs redevienne un sujet de débat public, sous l'influence du MEDEF et des débats sur la politique sociale européenne. Le RMI a fonctionné comme une soupape de sécurité et a épargné la misère absolue aux chômeurs en fin de droits, mais il n'a en rien contribué à la formation des chômeurs. L'insertion est en fait souvent très éloignée des demandes du marché du travail, n'est proposée qu'à une petite minorité de RMIstes, et revêt un caractère facultatif.

8 L'originalité du RMI par rapport aux politiques britanniques est donc très relative, si elle tient uniquement à la notion d'insertion, qui ne recouvre pratiquement aucune réalité. L' Income Support et avant 1984 le Supplementary Benefit, et avant Harold Wilson la National Assistance représentaient des allocations différentielles, qui complétaient les revenus des allocataires jusqu'à un seuil minimal, ce qui est exactement le principe du RMI. ${ }^{10}$

Enfin la France d'avant 1990 a recours à un troisième type de politique, qui consiste à repousser l'entrée des jeunes mal formés sur le marché du travail. Des pactes pour l'emploi de 1978 aux TUC, aux contrats en alternance, aux crédits formation et aux CES, tout un arsenal a été créé, intégrant dans une proportion variable deux ingrédients supplémentaires, la formation et l'abaissement du coût du travail. ${ }^{11}$ Fondamentalement, ces programmes ne diffèrent que peu des Youth Opportunity Programmes et des Youth Training Schemes britanniques. ${ }^{12}$

10 A partir du début des années 1990, la politique française va consister de plus en plus à abaisser le coût du travail, et à réduire la durée hebdomadaire de celui-ci. Du point de vue des emplois aidés, il n'y a pas solution de continuité avec les politiques précédentes, mais accélération de celles-ci. Le développement des CES et des emplois jeunes dans le secteur non marchand permet aux services publics des embauches qui seraient impossibles dans le cadre réglementaire normal, du fait de leur coût. De nombreuses dispositions sont prises pour alléger les cotisations sociales pour les smicards à partir de 1993, et pour les emplois familiaux et les services aux personnes (chèques emploi service). Enfin, des abattements sont consentis sur les charges patronales pour les emplois à temps partiel. ${ }^{13}$ L'abaissement du coût du travail était sans nul doute un des objectifs des thatchériens des années 1980. Là où ces derniers comptaient sur la loi d'airain du marché du travail pour amener les demandeurs d'emploi à plus de réalisme, et n'hésitaient pas à affaiblir le syndicalisme pour l'empêcher d'interférer avec le marché, les Français ont préféré offrir aux employeurs des avantages considérables, et faire prendre en charge graduellement par la nation une part de plus en plus importante du financement de la protection sociale. La méthode est différente, mais l'objectif est le même.

11 La question de la réduction du temps de travail s'inscrit partiellement dans une logique de lutte contre le chômage. Il convient d'être très prudent sur cette question, car les 35 heures ont été justifiées au nom de principes très différents selon le public auquel s'adressaient les discours officiels. Il est donc difficile de démêler l'écheveau, et de distinguer la rhétorique des réelles motivations des politiques publiques. Le mouvement syndical a lui-même été divisé sur la question pendant les années 1980, certains, du côté du socialisme chrétien, acceptant l'idée d'un partage du travail avec baisse des revenus, de façon à inclure les chômeurs, d'autres tenant à une compensation salariale intégrale, d'autres encore affichant leur scepticisme et donnant la priorité au maintien du pouvoir d'achat. Le bilan du passage aux 39 heures, en 1982, est officiellement de 140000 emplois 
créés, chiffre important qui ne fait pas l'unanimité. ${ }^{14}$ La capacité des 35 heures à créer des emplois est un sujet de controverse, dans une société de services, dans laquelle la capacité de calcul des microprocesseurs double tous les 18 mois. Même si l'efficacité de tous les services de secrétariat ne s'améliore pas au même rythme, l'augmentation de la productivité permet peut-être, dans certains cas, d'absorber une diminution du temps de travail sans embauches. L'impact des 35 heures, dont on commence juste à prendre la mesure, est considérable en termes de rythmes de vie, d'organisation des loisirs, mais aussi de stress et de flexibilisation de l'organisation du travail. Cette politique de réduction du temps de travail est, apparemment, sans équivalent en Grande-Bretagne.

Contrairement à la Grande-Bretagne de Blair, la France n'est pas parvenue dans la même période à faire baisser le niveau de chômage de façon réellement significative, malgré une embellie en 1999-2000. La brutalité légendaire et l'efficacité de la main invisible ne sont plus à démontrer.

\section{Les chemins de la flexibilité}

13 Les politiques publiques employées par les deux pays donnent souvent lieu à des comparaisons qui sont, pour chacun, autant d'occasions de justifier sa propre politique. En fait, une analyse permet de mettre en lumière à la fois des divergences, dues pour l'essentiel à des pesanteurs historiques et culturelles, et de réelles convergences politiques. Nous prendrons les deux exemples de la flexibilité et de l'employabilité.

En matière de flexibilité, l'image d'Épinal, qui n'est pas totalement sans rapport avec la réalité, est connue. La France serait le pays du statut, lié à un niveau de qualification reconnu, et garanti par l'État. La flexibilité, tant interne qu'externe serait donc très faible. La Grande-Bretagne, à l'inverse, serait un pays où la flexibilité du marché du travail est maximale, comme en atteste le nombre de travailleurs à temps partiel, et l'évolution des salaires. ${ }^{15}$ Il est difficile de distinguer le discours de la réalité, car nous n'appréhendons cette dernière qu'à travers le premier. L'évolution des discours est effectivement spectaculaire. Dans la Grande-Bretagne pré-thatchérienne des années 1970, les « demarcation disputes» entre différentes catégories de travailleurs syndiqués étaient présentées comme monnaie courante, et les relations professionnelles comme extrêmement rigides, le "shop steward » honni étant responsable du "wage drift », depuis les années 1960. Le décalage entre France et Grande-Bretagne est donc peut-être simplement temporel, et lié à la plus grande rapidité du passage du fordisme au postfordisme en Grande-Bretagne. Le résultat aujourd'hui est le même, la proportion d'employés du tertiaire étant identique dans les deux pays (environ $70 \%) .{ }^{16}$ Dire que la méthode et les conditions du passage d'un système économique à un autre importent moins que le résultat n'est pas faux, mais un peu partial, car, du point de vue de l'âne, il y a une différence entre la carotte et le bâton, même si cela revient au même pour son propriétaire.

15 En termes de méthode, la France, dont les élites politico-économiques sont convaincues des bienfaits, ou au moins de l'inéluctabilité, de la nouvelle organisation de l'économie, ${ }^{17}$ a utilisé la réduction du temps de travail pour tenter d'augmenter la flexibilité interne des entreprises. Ceci n'affecte en rien la question de la flexibilité externe, sujet extrêmement politique qui s'est polarisé après 1986 sur la question de l'autorisation administrative de licenciement. La rapidité de l'évolution du marché du travail a permis, en douceur, une augmentation de la proportion des CDD par rapport aux CDI parmi les 
créations d'emplois. L'objectif affiché par Martine Aubry et inscrit dans la loi elle-même était de permettre une relance de la négociation collective sur l'organisation interne du travail, à l'occasion du passage aux 35 heures. En pratique, les négociations ont été extrêmement délicates, non pas tant par leurs aspects financiers que du fait de leurs implications pour l'ensemble des conditions de travail. Les thèmes du stress et du " harcèlement moral », c'est-à-dire les pressions exercées par le collectif de travail, ont fait leur apparition non seulement dans les gazettes, mais aussi dans les prétoires à partir de 1999. L'État a pris à sa charge une partie des coûts du passage aux 35 heures, remettant à plus tard le règlement de la facture par le budget, porté par l'espoir que les retombées économiques de la flexibilité auraient un effet positif sur les rentrées fiscales, à long terme. La question de la flexibilité est donc au cœur des relations professionnelles françaises, elle est un objet permanent de négociation, contrairement à ce qui est souvent affirmé.

Les différences concrètes résident en partie dans la plus grande généralisation du travail à temps partiel en Grande-Bretagne qu'en France, surtout pour les femmes (16\% au total en France, dont $29,5 \%$ pour les femmes, contre $24 \%$ au total en GB, dont $44,8 \%$ pour les femmes), alors que la France utilise plus les CDD que la GB (12,6\% contre 7,1\%). ${ }^{18}$ Ceci tendrait à montrer que la France ne répugne pas à recourir à la flexibilité externe, qui est la forme la plus brutale et la plus inquiétante du point de vue des droits sociaux et des conditions de vie. ${ }^{19}$

17 Globalement, c'est à l'extension des temps partiels que la Grande-Bretagne doit l'essentiel de la baisse du chômage au cours de la première moitié des années 1990, et les créations ne repartent à la hausse qu'à la fin de la décennie. En effet, le solde des emplois créés est faible : seulement $+1,7 \%$ entre 1979 et 1995 (contre $+26,4 \%$ aux États-Unis). ${ }^{20}$

Le mythe du travail à domicile et du travailleur indépendant, ultra-flexible, recevant dans sa demeure rurale branchée les commandes de ses clients sur son ordinateur personnel, effectuant le travail entre une promenade avec ses chiens et une séance de yoga, est du domaine du fantasme journalistique. Entre 1991 et 2000, le nombre de travailleurs indépendants en Grande-Bretagne baisse de 3,4 millions à 3,1 millions. Le travail à domicile passe de $3 \%$ à $2 \%{ }^{21}$

Enfin, une analyse du rapport entre flexibilité et chômage ne peut passer sous silence les implications politiques et sociales de la question, qui ne sont pas réductibles à un simple problème économique. Dans des sociétés démocratiques et évoluées, les agents économiques ont une perception aiguë de ces problèmes. En termes de droits, la flexibilité interne et surtout externe met en cause les notions de sécurité des individus et de respect de l'identité professionnelle. ${ }^{22}$ La garantie des droits sociaux, dans le domaine de la santé et des retraites, est indissociable d'une certaine stabilité. ${ }^{23}$ La sécurité est devenue partie intégrante des droits sociaux. En France, où la politique sociale est conçue comme une "protection" contre la dureté de l'économie, ce phénomène est particulièrement sensible, et l'État est sommé de jouer son rôle ${ }^{24}$. Ni la société britannique, ni la société française n'ont donc encore trouvé leur équilibre en la matière, et l'interprétation politique de la thématique de la flexibilité est encore dans les limbes. Une critique sociale radicale s'est fait jour en France, mettant en cause tant l'évolution du salariat, avec Robert Castel, ${ }^{25}$ que le «nouvel esprit du capitalisme » avec Boltanski et Chiappello ou Perrilleux. ${ }^{26}$ Par-delà la description des phénomènes et les débats académiques, on assiste à la mise en place de discours théoriques susceptibles de prendre 
la place laissée vacante par l'effacement du marxisme, et par l'éloignement de la perspective messianique de la prise du pouvoir par la classe ouvrière.

\section{L'« employabilité », un souci partagé}

La question de l'employabilité a été érigée au rang de principe par Tony Blair. La politique du New Labour, illustrée par les différents New Deals, doit son originalité à deux facteurs. Contrairement aux politiques d'insertion et de formation françaises, elle a un caractère contraignant, voire pratiquement obligatoire. Un jeune qui passe sous le portique magique du New Deal Gateway n'a d'autre choix que le travail ou la formation. De ce point de vue, on est relativement proche des politiques recommandées aux États-Unis, mais jamais systématisées dans ce pays pour des raisons de budget et de logistique, et prônées par des gens comme Lawrence Mead (Mead 1997, Rees 2000). ${ }^{27}$ En revanche, contrairement au workfare américain, qui a un côté plutôt punitif et considère n'importe quelle tâche comme rédemptrice en elle-même, le New Deal met l'accent sur la formation des chômeurs. Les coûts budgétaires du New Deal sont extrêmement élevés. ${ }^{28}$ Il est financé grâce au produit de certaines privatisations, et représente donc un pari historique plus qu'une politique à long terme, car il n'est ni reproductible à l'infini, ni soutenable pendant une très longue période. Le pari est fondé sur l'espoir que, grâce à cet électrochoc, le système de formation de la Grande-Bretagne s'améliore structurellement.

21 Le caractère obligatoire de la formation en Grande-Bretagne représente effectivement une différence significative avec la France, même si les conditions d'attribution des allocations de chômage ont été durcies considérablement à partir de 1993 dans ce pays, et si les discours officiels font une certaine place à la lutte contre la fraude. Il y a à cela au moins trois raisons.

La première est budgétaire. Si la notion d'insertion devait être prise au sérieux, le nombre de stages et de formations à mettre en place pour les chômeurs et les RMlistes demanderait une mobilisation humaine et budgétaire considérable. La seconde est administrative. La séparation fonctionnelle entre les ASSEDIC et l'ANPE rend encore plus difficile l'insertion des chômeurs. La troisième est culturelle. Certains pays, comme les pays scandinaves, voient la population intérioriser les lois. La pression sociale est alors très forte sur les citoyens pour qu'ils se conforment aux règles établies en commun. La France n'est pas le plus anarchique des pays, mais le respect des lois y est subordonné à un accord politique de la part des citoyens, et l'autorité de l'État n'est respectée que lorsqu'elle est considérée comme légitime, ce qui n'est pas toujours le cas. Nous sommes donc dans un cas de figure très différent de la Grande-Bretagne, où le respect de la règle, the rule of law, est considéré comme un des principes de base de la démocratie, et le premier critère de civilisation. Pour que la formation des chômeurs prenne un caractère obligatoire, et que ces dispositions soient effectivement respectées, il serait nécessaire, en France, de passer par l'intermédiaire de la politique, et peut être par une crise, ce mode de régulation favori du pays. C'est précisément ce que les gouvernants tentent d'éviter. A l'occasion du débat lancé par le MEDEF sur la «refondation sociale», la CFDT et le patronat seuls sont parvenus à un accord sur le PARE, c'est-à-dire une disposition visant à offrir à certains chômeurs une formation professionnelle supplémentaire, sanctionnée par des allocations plus généreuses. Ceci a été en partie repris à son compte par le gouvernement, mais en maintenant l'aspect facultatif, volontaire, de l'opération. La négociation sociale "à froid", en l'absence de crise et de forte implication des acteurs 
politiques, débouche donc sur un résultat très modeste. On assiste simplement à une mobilisation rhétorique intéressante, à usage européen, destinée à présenter la France comme en phase avec les politiques modernes de l'emploi, dites «active labour market policies». Des dispositions inspirées des préretraites, comme l'ARPE, destinées à remplacer les travailleurs âgés par des jeunes, ou les emplois jeunes sont ainsi décrits comme des " politiques actives », ce qui est un usage discutable du terme.

Sur le plan de la formation, la Grande-Bretagne a effectivement une longueur d'avance du point de vue de la formation continue, inventée avant même la Première Guerre mondiale, et organisée sous l'égide des syndicats ouvriers. Il n'y a pas d'équivalent en France à la Workers Educational Association, ni à Ruskin College. L'idée que des syndicats puissent repérer un jeune et talentueux militant, l'envoyer à l'université faire des études d'économie, et en faire un Premier ministre est impensable en France, où même les trotskistes ont pu considérer l'École nationale d'administration comme la voie royale pour accéder au pouvoir. Richard Hoggart, chantre de la culture ouvrière en péril dès 1957, plaçait la volonté d'apprendre, de s'améliorer, au centre de la démarche collective de la classe ouvrière, et de son éthique, et considérait la formation continue comme un des acquis du mouvement. ${ }^{29} \mathrm{~L}^{\prime}$ Open University, conçue dans cette perspective par ses créateurs travaillistes, a su allier la maîtrise des technologies de communication les plus modernes à l'excellence universitaire. Cet établissement, très important numériquement dans le pays, s'est adapté au zeitgeist libéral contemporain. Il est maintenant présent sur le marché international de la formation, et vient, jusque dans nos banlieues, éduquer nos fils et nos compagnes.

En revanche, si on prend en considération la formation initiale, on constate que la Grande-Bretagne a un important retard sur ses voisins, qui explique peut-être le souci des gouvernements Blair d'améliorer les choses. En effet, à l'âge de 18 ans, seuls $49 \%$ des Britanniques sont encore dans le système éducatif, contre $86 \%$ des Allemands, $69 \%$ des Français, $78 \%$ des Hollandais, $95 \%$ des Suédois. ${ }^{30}$ Les statistiques selon l'origine ethnique, dont les Britanniques sont friands, nous montrent que ce sont les Blancs qui quittent le système scolaire le plus tôt : dès l'âge de 16 ans, $15 \%$ des Blancs, contre seulement $5 \%$ des Indiens, et $10 \%$ des Noirs quittent le système scolaire. ${ }^{31}$ Ce choix n'est donc pas une conséquence de la pauvreté, mais est peut-être à rapprocher de la culture industrielle traditionnelle, dans laquelle une entrée rapide dans l'industrie était plus rentable que des études. S'appliquait ainsi le principe, à l'opposé de l'élitisme républicain, selon lequel «celui qui peut travailler travaille, celui qui ne peut pas trouver un emploi fait des études ». Le taux de scolarisation des régions industrielles de France était, il y a trente ans encore, sensiblement inférieur à celui des régions qui l'étaient moins. Dans un contexte qui laisse de moins en moins de place aux non qualifiés, et où les connaissances nécessitent un solide socle de compétences de base pour être mises à jour constamment, les sorties précoces du système scolaire sont un vrai problème. L 'obsession britannique de l'« employabilité » doit donc peut-être autant au poids du passé industriel qu'au souci de rendre les jeunes et les chômeurs industrieux.

Dans l'imaginaire collectif de la France, l'éducation joue un rôle de premier plan, et les comparaisons internationales suggèrent que la formation initiale est relativement efficace. Il n'y a donc pas d'obstacle à considérer la formation comme un devoir national, c'est-à-dire à la fois un droit et une obligation. Seules font débat la place relative de la formation continue et de la formation initiale, et l'importance du secteur privé. L' " employabilité » n'est donc pas un concept qui oppose totalement les deux pays : l'accent 
mis sur la formation n'est pas problématique sur le plan des principes, et seul le caractère obligatoire de celle-ci le reste, pour l'instant. En revanche, l'idée que l'employabilité doive remplacer le statut et la stabilité, et que seules les compétences du travailleur mobile et flexible puissent un jour garantir son niveau de vie, n'est pas acceptée en France.

\section{Conclusion}

La comparaison nous incite donc à la plus grande prudence par rapport aux oppositions rapides, et au besoin d'auto-justification des deux sociétés. Si les termes employés sont parfois différents, si les structures ne sont pas toujours les mêmes, les politiques publiques ont en fait des objectifs très proches. Les blocages se situent simplement parfois à des endroits différents, et nécessitent des approches spécifiques. Dans la mesure où les situations concrètes des deux pays sont beaucoup plus semblables aujourd'hui que par le passé, le seul facteur de divergence important est en fait de nature idéologique et culturelle, comme par exemple l'influence du libéralisme sur la totalité de l'éventail politique britannique et le rapport français à l'État. La Grande-Bretagne s'est rendue célèbre en Europe pour son opposition à la politique sociale européenne. Margaret Thatcher en son temps refusait de signer la charte sociale et Tony Blair a pris soin à plusieurs reprises d'éviter toute disposition qui viendrait contredire la flexibilité..$^{32} \mathrm{En}$ réalité, il serait bon de définir ce que l'on entend par politique sociale européenne. S'il s'agit de la politique de la Commission européenne, du Conseil des ministres et du Parlement européen, celle-ci est encore dans les limbes, et, pour l'essentiel à l'état de recommandation. La prudence britannique à son égard n'a donc strictement aucune importance, et les discours du gouvernement Blair relatifs à cette question en GrandeBretagne sont purement à usage interne. Il s'agit de tenir le langage qui rassurera les milieux dirigeants de l'économie britannique, et, par médias interposés, les citoyensélecteurs de la "classe moyenne " avant tout attachés à la santé de l'économie et au respect des règles du marché. En revanche, si par "politique sociale européenne », on entend la somme des politiques sociales menées en Europe, la Grande-Bretagne est loin d'être isolée. Elle est bel et bien "au cœur de l'Europe », selon le vœu de Tony Blair. ${ }^{33}$ L'idée selon laquelle il n'est pas de salut hors de la flexibilité et des politiques d'emploi fondées sur la formation des chômeurs plutôt que sur le versement d'allocations, les fameuses "active labour market policies" se sont imposées dans la plupart des pays européens, au moins chez les experts, même si elles sont difficiles à appliquer. Le modèle $\mathrm{du}$ " poldersocialisme » néerlandais a été l'exemple le plus spectaculaire de ce type de politique, en favorisant systématiquement le recours au travail à temps partiel. La France a fait une fois encore figure d'exception en choisissant, seule en Europe, la voie de la réduction du temps de travail, avec le double objectif du partage du travail et de l'amélioration de la flexibilité. Mais il s'agit, là encore, d'un choix tactique et non d'une divergence sur les objectifs. Le passage de la convergence sur les objectifs, et sur les concepts fondamentaux, qui est déjà pratiquement effectuée au niveau européen, à un accord sur les politiques publiques est en fait extrêmement difficile. Nous sommes pourtant en présence de politiques qui partagent toutes des caractéristiques fortes, et très visibles pour peu qu'on les regarde de l'extérieur. La combinaison des capacités d'innovation technologique et du dynamisme économique du capitalisme d'une part, et des priorités traditionnelles de l'Europe en matière de formation et de garanties sociales de l'autre, constitue bien un modèle distinct de celui qui prévaut aux États-Unis, sans 
parler du reste du monde. Il y a bien un modèle européen, dont les politiques britannique et française sont deux versions, distinctes mais parfois moins éloignées qu'il n'y paraît.

\section{BIBLIOGRAPHIE}

ANDREWS, K. \& JACOBS, J. Punishing the Poor: Poverty under Thatcher. London: Macmillan, 1990. BLACKMAN, T. \& PALMER, A. 'Continuity or Modernization? The Emergence of New Labour's Welfare State'. Social Policy Review 11, 1999, p.106-126.

BLAIR, T. Britain's Role In Europe. Prime Minister's Speech to the European Research Institute, Birmingham, 23 $3^{\text {rd }}$ November 2001.

BOISSONNAT, J. Le Travail dans vingt ans. Paris : Odile Jacob, 1995.

BOLSTANSKI, L \& CHIAPPELLO, E. Le Nouvel esprit du capitalisme. Paris : Gallimard, 1999.

BOULLIER, K. Maîtriser la réforme des 35 heures. Paris : Éditions Liaisons, 1998.

BRYSON, A. \& JACOBS, J. Policing the Workshy. London: Avebury, 1990.

CASTEL, R. Les Métamorphoses de la question sociale. Paris : Fayard, 1995.

CHILDS, David. Britain since 1945: A Political History. London: Routledge, 1997.

CLASEN, J. (ed). Comparative Social Policy. Oxford : Blackwell, 1999.

Commissariat général au plan. Chômage : le cas français. Paris : La Documentation française, 1997.

EDWARDS, John \& RÉVAUGER, Jean-Paul (eds.). Employment and Citizenship in Britain and France. London: Ashgate, 2000.

FIELD, F. \& OWEN, M. Beyond Punishment: Hard Choices on the Road to Full Employability. London: Institute of Community Studies, 1994.

FRIOT, B. Puissances du salariat : Emploi et protection sociale à la française. Paris : La dispute, 1996. GEORGE, V. \& TALOR GOOBY, P. European Welfare Policy. London: Macmillan, 1996.

GLENNERSTER, H. ‘A Third Way?’ Social Policy Review 11, 1999, pp. 28-44.

Her Majesty's Treasury. Budget 2001. London: The Stationery Office, 2001.

HOGGART, R. The Uses of Literacy. London: Chatto \& Windus, 1957.

HUTTON, W. The Stakeholding Society. London: Polity Press, 1999.

JOHNSON, Christopher. The Economy under Mrs Thatcher. Harmondsworth: Penguin, 1991.

JOIN-LAMBERT, M.-T. Politiques sociales. Paris : Presses de Sciences Po et Dalloz, 1997.

MACNICOL, J. 'Dilemmas of Age Discrimination'. Communication présentée lors du colloque «Les discriminations : Analyse comparée de la France et de la Grande-Bretagne » Aix-en-Provence, Septembre 2000. Publication en cours.

MEAD, L. From Welfare to Work. London: Institute of Economic Affairs. 1997. 
MEYER, J.L. Des Contrats Emploi Solidarité aux Emplois Jeunes. Paris : L'Harmattan, 1999.

MINC, A. La France de l'An 2000. Rapport au Premier ministre. Commissariat général au plan. Paris : La Documentation française, 1994.

MIRE. Comparer les systèmes de protection sociale en Europe. Vol 2. Rencontres de Berlin. Paris : MIRE, 1996. ISBN 2-11-089005-3

National Statistics. Social Trends. London: The Stationery Office, 2001.

PÉRRILLEUX, T. Les Tensions de la flexibilité. Paris : Desclée de Brouwer, 2001.

PORTONNIER, J.-C. Glossaire bilingue de la protection sociale. Social protection : A bilingual glossary.

Paris : MIRE, Rencontres et Recherches, 1998.

RÉVAUGER, J.-P. ‘France Reduces the Working Week'. SPA News. Social Policy Association. Spring 1999.

Secretary of State for Education and Employment. Towards Full Employment in a Modern Society. London: The Stationery Office, 2001.

Secretary of State for Social Security and Minister for Welfare Reform. A New Contract for Welfare. London: The Stationery Office, 1998.

SKED, Alan \& COOK Chris. Post-War Britain, Harmondsworth: Penguin, 1979 (nombreuses rééditions).

WISE, M. \& GIBB, R. Single Market to Social Europe. London: Longman, 1993.

WUHL, S. Insertion: les politiques en crise. Paris : PUF, 1996.

\section{NOTES}

1. Dans un discours prononcé par le ministre travailliste Anthony Crosland à Manchester le 9 mai 1975. L'histoire a aussi retenu : 'We used to think we could spend our way out a crisis. That option is no longer open.' (James Callaghan, intervention au congrès du parti travailliste le 28 septembre 1976). Sur le contexte économique de l'époque, voir par exemple Alan SKED \& Chris COOK, PostWar Britain, Harmondsworth: Penguin, 1990 (3 ${ }^{\text {rd }}$ edition), p. 314.

2. David CHILDS, Britain since 1945: A Political History, London: Routledge, 1997, p. 214.

3. A. BRYSON \& J. JACOBS, Policing the Workshy, London: Avebury, 1990.

4. Sur le rapport entre le chômage et la fin du fordisme, voir la première partie du rapport du commissariat au plan (Jean BOISSONNAT, Le Travail dans vingt ans, Paris : Odile Jacob, 1995).

5. M.-T. JOIN-LAMBERT, Politiques sociales, Paris : Presses de Sciences Po et Dalloz, 1997, p. 220.

6. Ibid, p. 230.

7. John MACNICOL, "Dilemmas of Age Discrimination". Communication présentée lors du colloque "Les Discriminations: Analyse comparée de la France et de la Grande-Bretagne ", Aix-enProvence, septembre 2000, publication en cours.

8. Commissariat Général au Plan, Chômage : le cas français, Paris : La Documentation française, 1997.

9. Simon WUHL, Insertion : les politiques en crise, Paris : PUF, 1996.

10. On peut simplement noter qu'à partir de 1988 la Job Seekers Allowance excluait les chômeurs de l'Income Support .

11. Sur les emplois jeunes, voir l'ouvrage de Jean-Louis MEYER, Des contrats emploi-solidarité aux emplois jeunes, Paris : L’Harmattan, 1999. 
12. On peut simplement noter une différence importante entre les deux sociétés. La place du patronat dans l'éducation est un sujet particulièrement délicat et controversé en France, où l'éducation est présentée comme un des éléments constitutifs de la citoyenneté, et non simplement comme une formation permettant de donner aux travailleurs des compétences techniques. Même si nous restons dans le discursif, les idées ne sont pas sans conséquences. Par ailleurs, les jeunes travailleurs bénéficient des mêmes garanties que leurs aînés en matière de salaire minimum, le statut étant indépendant de l'âge. Un Premier ministre français, Edouard Balladur, paiera cher sa volonté d'introduire en France une réforme thatchérienne, le «smic jeune $"$.

13. M.-T. JOIN-LAMBERT , op.cit., p. 287 et suivantes.

14. Ibid., p. 290.

15. J.-P. RÉVAUGER, «Flexibility: in Search of a New Social Settlement » in John EDWARDS \& Jean-Paul RÉVAUGER (eds.), Employment and Citizenship in Britain and France, London: Ashgate, 2000.

16. Jean-Louis MEYER, op.cit., 1999, p. 36.

17. Jean BOISSONAT, op.cit., 1995 et Alain MINC (Rapport au Premier ministre de la commission présidée par), La France de l'An 2000, Paris : éditions Odile Jacob, La Documentation Française, 1994.

18. J.-L. MEYER, op. cit., p.43.

19. La prédominance du temps partiel en Grande-Bretagne est à mettre en regard de statistiques analysant la satisfaction au travail. Il apparaît que l'insatisfaction est plus forte, en GrandeBretagne, pour les travailleurs à temps plein que pour ceux qui sont à temps partiel. $60 \%$ des hommes et $63 \%$ des femmes employés à temps partiel s'estiment « satisfaits ou très satisfaits » de leur emploi, contre $49 \%$ et $56 \%$ pour les travailleurs à temps plein. Seuls $13 \%$ des hommes et $12 \%$ des femmes employés à temps partiel se disent insatisfaits ou très insatisfaits, contre $24 \%$ et $16 \%$ des salariés à temps plein (National Statistics, Social Trends, London: The Stationery Office, p. 90) . On pourrait donc suggérer que la flexibilité est un moyen de réduire l'insatisfaction, car elle entraîne une plus faible implication dans le travail. La différence entre les hommes et les femmes en Grande-Bretagne est spectaculaire, puisque l'emploi féminin est, dans $44 \%$ des cas, à temps partiel. Voilà en tout cas qui permet de nuancer les discours structuralistes d'inspiration weberienne sur l'éthique protestante du travail, qui semble surtout s'appliquer aux hommes.

20. M.-T. JOIN-LAMBERT, op.cit., p. 287.

21. National Statistics, op. cit., pp. 82-83.

22. David MIDDLETON, 'Labour Market Flexibility, Security and Self Respect', in John EDWARDS \& Jean-Paul RÉVAUGER (eds), op. cit.

23. John EDWARDS, 'Flexibility, Economic Security and Social Rights', in John EDWARDS \& JeanPaul RÉVAUGER (eds.), ibid.

24. En outre, la capacité à faire des prévisions est indispensable tant pour les stratégies immobilières et patrimoniales que pour les stratégies éducatives qui sont l'apanage des classes moyennes européennes, modèle d'identification proposé à l'ensemble de la population, et socle $\mathrm{du}$ « capitalisme populaire » comme de la méritocratie.

25. Robert CASTEL, Les Métamorphoses de la question sociale, Paris : Fayard, 1995.

26. L. BOLTANSKI \& E. CHIAPPELLO, Le Nouvel esprit du capitalisme, Paris : Gallimard, 1999. T. PERRILLEUX, Les Tensions de la flexibilité, Paris : Desclée de Brouwer, 2001.

27. Lawrence MEAD, From Welfare to Work, London: Institute of Economic Affairs, 1997.

28. Un total de 2890 millions de livres était prévu pour le New Deal entre 1997 et 2001 . Her Majesty's Treasury, Budget 2001, London: The Stationery Office, 2001.

29. Richard HOGGART, The Uses of Literacy, London: Chatto \& Windus, 1957.

30. National Statistics, op. cit., p. 65.

31. Ibid.

Revue Française de Civilisation Britannique, XII-2 | 2003 
32. Mark WISE \& Richard GIBB, Single Market to Social Europe, London: Longman, 1993. Tony BLAIR, Britain's Role in Europe, Birmingham: Prime Minister's Speech to the European Research Institute, $23^{\text {rd }}$ November 2001.

33. Ibid.

\section{RÉSUMÉS}

Les politiques d'emploi françaises et britanniques sont plus semblables qu'il n'y paraît. Le décalage est essentiellement temporel. Dès 1979, la Grande-Bretagne thatchérienne a utilisé à la fois l'aiguillon de la nécessité et les politiques publiques pour inciter les Britanniques à s'adapter à la nouvelle économie, et à entrer dans le post-fordisme, par le moyen de la flexibilité et des temps partiels. La France a utilisé dans un premier temps le traitement social (préretraites, RMI, emplois aidés), confié à la "solidarité » plus qu'à l'assurance. A partir de 1990, elle s'est aussi engagée dans une politique de baisse du coût du travail. Stratégiquement, dans les deux pays, les concepts structurants sont la flexibilité et l'employabilité, même si les moyens de les mettre en œuvre diffèrent.

Employment policies in Britain and France are more similar than one might think. The difference is more a question of timing than substance. From 1979, under the premiership of Mrs Thatcher, Britain has used both public policies and economic pressure in order to encourage the population to adjust to the New Economy, and to post-Fordism. Flexibility and part-time work were used to this end. For another ten years, until 1990, France resorted to the traditional 'social treatment' of unemployment, including early retirement, minimum incomes and subsidised jobs. This was increasingly financed by taxation, and the importance of the insurance-based unemployment benefits declined. After 1990, France adopted a policy consisting in reducing the cost of labour, similar to Britain's. In both countries, the key concepts are flexibility and employability, although the practical policies used to implement them are different.

\section{AUTEUR}

\section{JEAN-PAUL RÉVAUGER}

Université Michel de Montaigne Bordeaux 3 